$$
\begin{array}{rlrl}
v^{(7)}= & D^{7}\left[-i\left\{\left(\frac{35}{64}+\frac{35}{4 B^{2}}+\frac{63}{4 B^{4}}+\frac{1}{B^{6}}\right) \cos p t\right.\right. & \left.\left.+\left(\frac{35}{16 B}+\frac{525}{8 B^{3}}\right) \sin 5 p t+\frac{7}{16 B} \sin 7 p t\right\}\right] v^{(0)} \\
& +\left(\frac{21}{64}+\frac{35}{2 B^{2}}+\frac{301}{4 B^{4}}\right) \cos 3 p t+\left(\frac{7}{64}+\frac{35}{4 B^{2}}\right) \cos 5 p t & v^{(9)}= & -D^{9}\left[\left(\frac{63}{16 B}+\frac{1575}{16 B^{3}}+\frac{735}{2 B^{5}}+\frac{255}{2 B^{7}}\right) \sin 2 p t\right. \\
& \left.\left.+\frac{1}{64} \cos 7 p t\right\}\right] v^{(0)} & +\left(\frac{63}{16 B}+\frac{693}{4 B^{3}}+\frac{3885}{4 B^{5}}\right) \sin 4 p t \\
v^{(8)}= & D^{8}\left[\left(\frac{35}{128}+\frac{35}{8 B^{2}}+\frac{63}{8 B^{4}}+\frac{1}{2 B^{6}}\right)\right. & +\left(\frac{27}{16 B}+\frac{1323}{16 B^{3}}\right) \sin 6 p t+\frac{9}{32 B} \sin 8 p t \\
& +\left(\frac{7}{16}+\frac{315}{16 B^{2}}+\frac{231}{2 B^{4}}+\frac{127}{2 B^{6}}\right) \cos 2 p t & -i\left\{\left(\frac{63}{128}+\frac{525}{32 B^{2}}+\frac{735}{8 B^{4}}+\frac{255}{4 B^{6}}+\frac{1}{B^{8}}\right) \cos p t\right. \\
& +\left(\frac{7}{32}+\frac{189}{8 B^{2}}+\frac{1701}{8 B^{4}}\right) \cos 4 p t+\left(\frac{1}{16}+\frac{133}{16 B^{2}}\right) \cos 6 p t & +\left(\frac{21}{64}+\frac{1071}{32 B^{2}}+\frac{7035}{16 B^{4}}+\frac{3025}{4 B^{6}}\right) \cos 3 p t \\
& +\frac{1}{128} \cos 8 p t+i\left\{\left(\frac{35}{16 B}+\frac{105}{4 B^{3}}+\frac{63}{2 B^{5}}+\frac{1}{B^{7}}\right) \sin p t\right. & +\left(\frac{9}{64}+\frac{861}{32 B^{2}}+\frac{6951}{16 B^{4}}\right) \cos 5 p t \\
+ & +\left(\frac{63}{16 B}+\frac{735}{8 B^{3}}+\frac{483}{2 B^{5}}\right) \sin 3 p t & \left.\left.+\left(\frac{9}{256}+\frac{231}{32 B^{2}}\right) \cos 7 p t+\frac{1}{256} \cos 9 p t\right\}\right] v^{(0)} .
\end{array}
$$

\title{
Input Impedance of Wide-Angle Conical Antennas Fed by a Coaxial Line*
}

\author{
CHARLES H. PAPAS $\dagger$, ASSOCIATE, IRE, AND RONOLD KING†, SENIOR MEMBER, IRE
}

Summary-The input impedances for conical antennas fed by a coaxial line have been computed for several flare angles. A graph of the auxiliary functions $\zeta_{n}(x)$ is included to facilitate impedance calculation for any large flare angie.

\section{INTRODUCTION}

I

N THE FALL of 1946, L. Brillouin suggested the problem of determining the input impedance of a spherically capped conical antenna fed by a coaxial line. This paper deals with only those aspects of the solution which are of immediate engineering interest. A complete theoretical discussion of this problem ${ }^{1}$ and a closely related one ${ }^{2-4}$ are found elsewhere.

* Decimal classification: R326.612 $\times$ R221. Original manuscript received by the Institute, March 15, 1949; revised manuscript received June 9, 1949. The research reported in this document was made possible through support extended Cruft Laboratory, Harvard University, jointly by the U. S. Navy Department (Office of Naval Research), the Signal Corps of the U. S. Army, and the U. S. Air Force, under ONR Contract N5ori-76, T. O. I.

$\dagger$ Cruft Laboratory, Harvard University, Cambridge, Mass.

${ }^{1}$ C. H. Papas and R. King, "Input impedance of wide-angle conical antennas," Technical Report No. 52, Cruft Laboratory, Harvard University.

${ }^{2}$ P. D. P. Smith, "The conical dipole of wide angle," Jour. Appl. Phys., vol. 19, pp. 11-23; January, 1948.

${ }^{3} \mathrm{C}$. T. Tai, "Application of variational principle to the study of biconical antennas," Technical Report No. 75, Cruft Laboratory, Harvard University.

'S. A. Schelkunoff, "Electromagnetic Waves," D. Van Nostrand Co., Inc., New York, N. Y., 1943; p. 472-473.
Specifically, the problem consists of determining the impedance which a wide-angle conical antenna of length $a$ and flare angle $\theta_{0}$ presents to a coaxial line with an infinite flange (Fig. 1). Its solution is formulated by con-

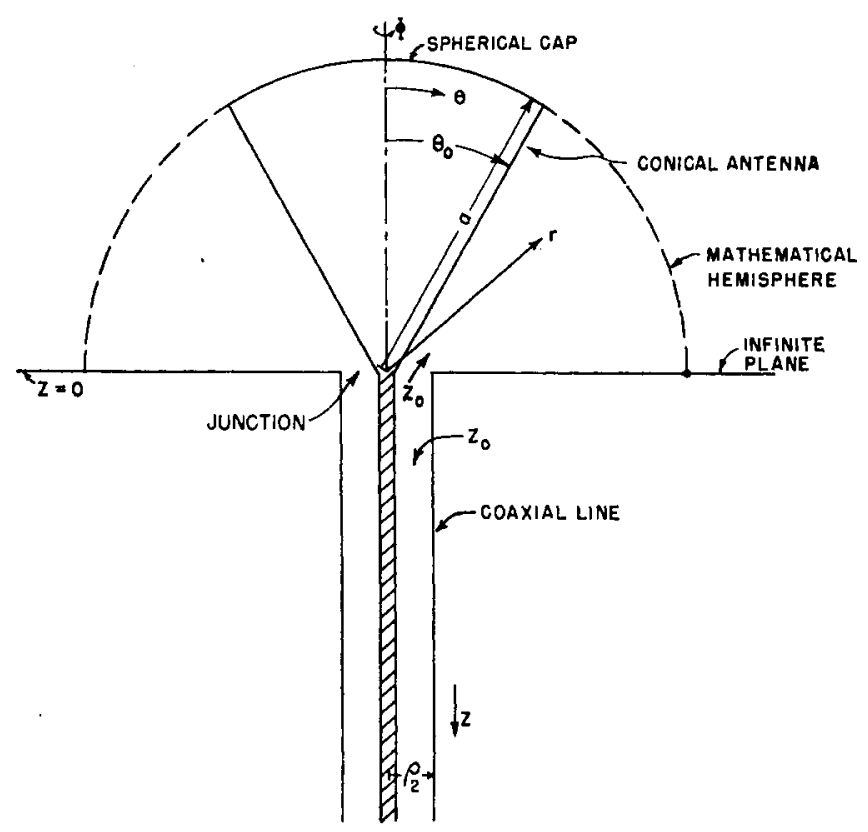

Fig. 1-Spherically capped conical antenna fed by coaxial feed line. 
sidering two regions: the antenna region, $\rho_{2} \leqq r \leqq a$, $\theta_{0} \leqq \theta \leqq(\pi / 2)$, and the radiation region $r \geqq a, \leqq 0 \theta \leqq(\pi / 2)$. The nonvanishing components $H_{\phi}, E_{\theta}$, and $E_{\mathrm{r}}$ of the magnetic- and electric-field vectors are expanded in series of eigenfunctions appropriate to the two regions and the unknown constants of the expansions are determined by forcing the anninilation of $E_{\theta}$ over the spherical cap and the continuity of $H_{\phi}$ and $E_{\theta}$ across the mathematical surface $r=a, \theta_{0} \leqq \theta \leqq(\pi / 2)$. At the junction between the antenna and line, the higher nonpropagating modes are neglected from the start, but since it is assumed that the characteristic impedance of the antenna is equal to that of the line, the difference between the input impedance of the antenna and the equivalent impedance which the antenna presents to the end of the line is capacitive and assumed to be negligibly small. The formal solution finally appears in the form of an infinite set of simultaneous equations. The equations are quite unmanageable, but when it is noticed that, for large values of flare angle, the complementary modes in the antenna region can be neglected in comparison to the TEM modes (one outwardly propagating, the other reflected from the surface $r=a$, $\left.\theta_{0} \leqq \theta \leqq(\pi / 2)\right)$, the problem reduces to the evaluation of the reflection coefficient of the $T E M$ wave.

\section{IMPEDANCE FORMULA}

Within the limits of the above-mentioned approximations, it is found that the input impedance of an antenna ${ }^{1}$ of length $a$ and flare angle $\theta_{0}$ is given by

$$
Z_{\text {in }}=Z_{0} \frac{1-\beta / \alpha}{1+\beta / \alpha}
$$

where

$$
\begin{aligned}
& Z_{0}=60 \ln \cot \frac{\theta_{0}}{2}, \\
& \frac{\beta}{\alpha}=e^{-2 i k a} \frac{1+i \frac{60}{Z_{0}} \sum_{n=1}^{\infty} \frac{2 n+1}{n(n+1)}\left[P_{n}\left(\cos \theta_{0}\right)\right]^{2} \zeta_{n}(k a)}{-1+i \frac{60}{Z_{0}} \sum_{n=1}^{\infty} \frac{2 n+1}{n(n+1)}\left[P_{n}\left(\cos \theta_{0}\right)\right]^{2} \zeta_{n}(k a)},
\end{aligned}
$$

and

$$
\zeta_{n}(k a)=\frac{h_{n}{ }^{(2)}(k a)}{h_{n-1}{ }^{(2)}(k a)-\frac{n}{k a} h_{n}{ }^{(2)}(k a)}
$$

$h_{n}{ }^{(2)}$ is the spherical Hankel function of the second kind, and $P_{n}(\cos \theta)$ is the Legendre polynomial of order $n$. The summation in (3) is over odd integral values. $Z_{0}$ is called the characteristic impedance of the antenna, $\beta / \alpha$ is the ratio of the amplitudes of the reflected and outwardly propagating $T E M$ waves in the antenna region, and $\zeta_{n}(k a)$ is a complex auxiliary function of the real variable $k a$ ( $k a=2 \pi \div$ free-space wavelength). It should be emphasized that (1) accurately predicts the equiva- lent impedance which the antenna presents to the end of the coaxial line only when the characteristic impedance of the feeding coaxial line; i.e., $60 \ln \rho_{2} / \rho_{1}$ where $\rho_{2}$ and $\rho_{1}$ are the radii of its outer and inner conductors, is made equal to the characteristic impedance of the antenna (2), and the flare angle is large. It is clear that there must be an upper bound to the admissable flare angles since, as the flare angle increases, the bend at the

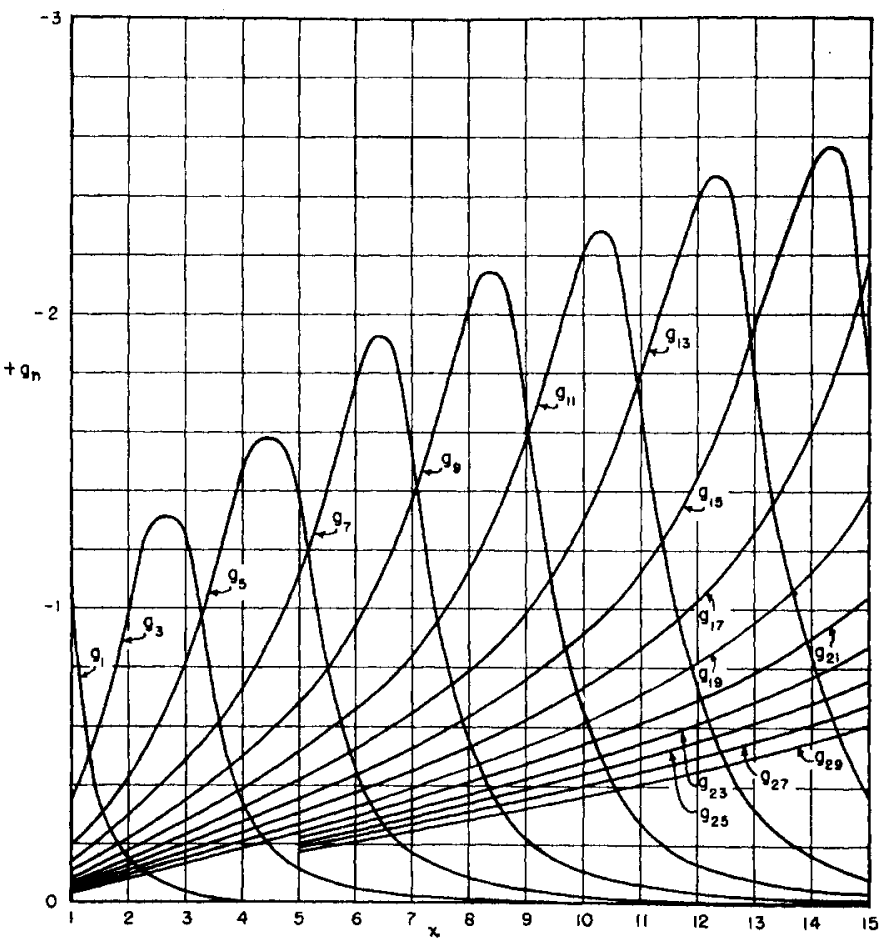

Fig. 2-Real part of the zeta function, $g_{n}(x)$ versus $x$.

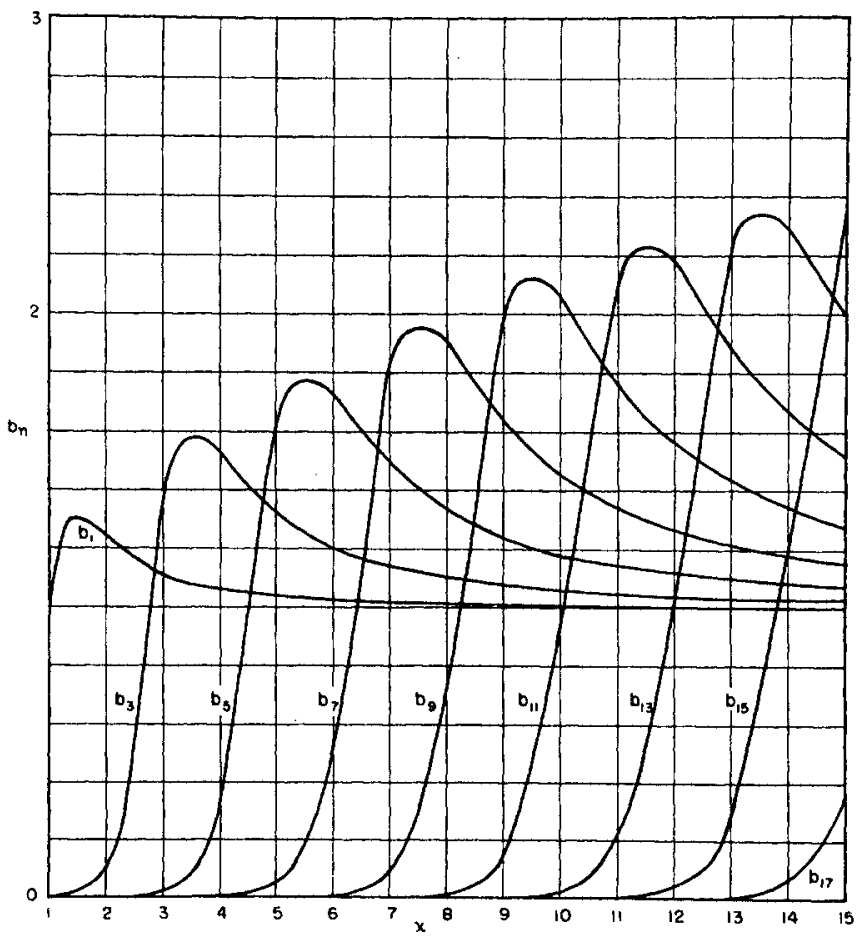

Fig. 3-Imaginary part of the zeta function $b_{n}(x)$ versus $x$. 
junction becomes more severe thereby making the higher nonpropagating modes there far from negligible. From experimental results (Fig. 4), it is safe to choose $30^{\circ}$ as the lower bound for $\theta_{0}$.

\section{REsults}

Using (4) the auxiliary functions $\zeta_{n}(x)=g_{n}(x)+i b_{n}(x)$ have been computed for various values of $n$ and $x$ (Figs. 2 and 3 ). With the aid of these computed functions, $\beta / \alpha$ and $Z_{0}$ can be evaluated for an antenna with length $k a$ and flare angle $\theta_{0}$ from (3) and (2) respectively. Substituting the values of $\beta / \alpha$ and $Z_{0}$ thus obtained into (1) yields the input impedance as a function of $\theta_{0}$ and $k a$.

For $\theta_{0}=30^{\circ}, 40^{\circ}, 55^{\circ}, 70^{\circ}$, the input impedances were computed for values of $k a$ ranging from 0 to 8 (Figs. $4,5,6$, and 7$)$. It is seen that the input resistance curves

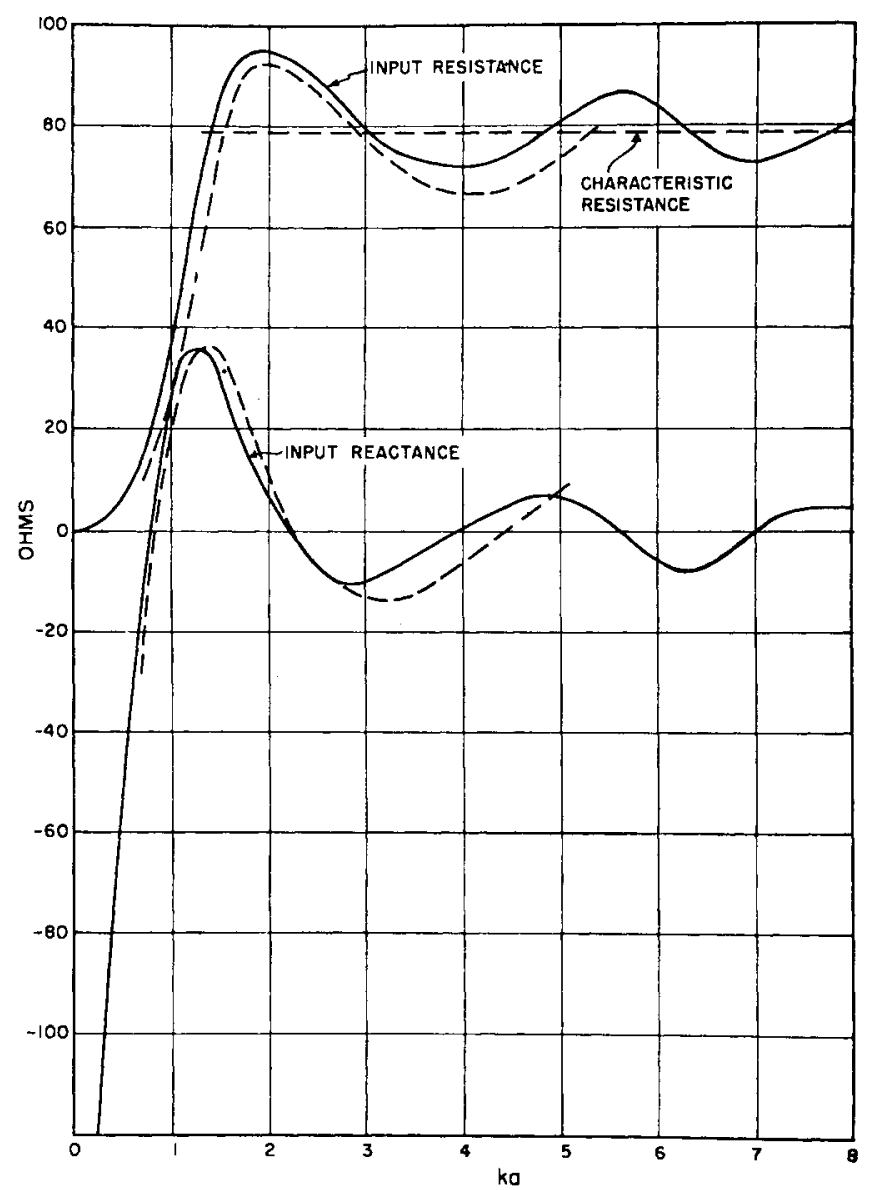

Fig. 4-Input resistance and reactance versus the parameter $k a$ for $\theta_{0}=30^{\circ}$, solidline indicates calculated values; broken line, measured values.

rise from zero and perform damped oscillations about the characteristic resistances, and the imput reactance curves start at large negative values and then perform damped oscillations about zero ohms. For $\theta_{0}=30^{\circ}$ the remarkably close agreement between the calculated and measured impedance ${ }^{5}$ is seen.

\section{ACKNOWLEDGMENT}

The authors wish to thank C. T. Tai for many valuable discussions, and also Mrs. R. Stokey, who performed all the computations.

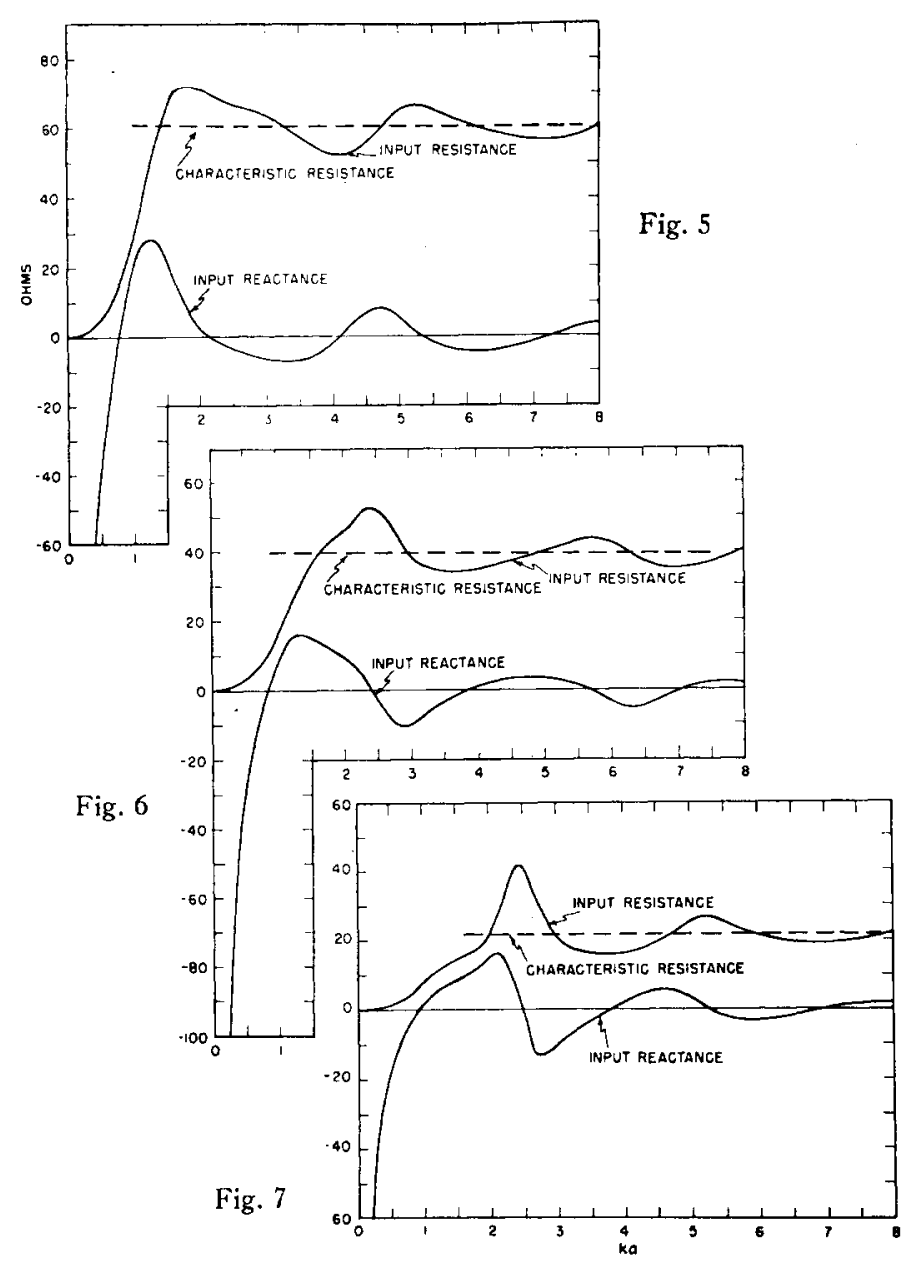

Fig. 5-Input resistance and reactance versus the parameter $\mathrm{ka}$ for $\theta_{0}=40^{\circ}$.

Fig. 6-Input resistance and reactance versus the parameter for $\theta_{0}=55^{\circ}$.

Fig. 7-Input resistance and reactance versus the parameter for $\theta_{0}=70^{\circ}$.

- Harvard Radio Research Laboratory Staff, "Very High, Frequency Techniques," McGraw-Hill Book Co., Inc., New YorkN. Y., 1947; vol. 1, p. 103, Fig. 4-10(d).

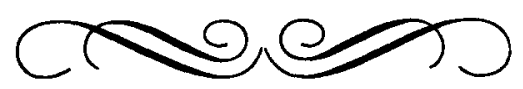

